\title{
NEW APPLICATIONS OF EXTREMELY REGULAR FUNCTION SPACES
}

\author{
TROND A. ABRAHAMSEN, OLAV NYGAARD, AND MÄRT PÕLDVERE
}

\begin{abstract}
Let $L$ be an infinite locally compact Hausdorff topological space. We show that extremely regular subspaces of $C_{0}(L)$ have very strong diameter 2 properties and, for every real number $\varepsilon$ with $0<\varepsilon<1$, contain an $\varepsilon$-isometric copy of $c_{0}$. If $L$ does not contain isolated points they even have the Daugavet property, and thus contain an asymptotically isometric copy of $\ell_{1}$.
\end{abstract}

\section{INTRODUCTION}

Throughout, let $L$ be an infinite locally compact Hausdorff topological space, and denote as usual by $C_{0}(L)$ the Banach space of continuous $\mathbb{K}$ valued functions on $L$ that "vanish at infinity", where $\mathbb{K}$ is the field of either real or complex numbers.

Definition 1.1 (Cengiz [7]). An extremely regular function space is a linear subspace $\mathcal{A}$ of $C_{0}(L)$ such that for every $x_{0} \in L$, every real number $\varepsilon$ with $0<\varepsilon<1$, and every open neighbourhood $V$ of $x_{0}$, there exists an $f \in \mathcal{A}$ such that

$$
\|f\|=1=f\left(x_{0}\right)>\varepsilon>\sup _{x \in L \backslash V}|f(x)| .
$$

The interest in extremely regular function spaces came from their importance in Banach-Stone type theorems. An example, also due to Cengiz [7], is as follows: If $L_{1}$ and $L_{2}$ are locally compact Hausdorff topological spaces such that there exists a linear isomorphism $\varphi$ from an extremely regular subspace of $C_{0}\left(L_{1}\right)$ onto such a subspace of $C_{0}\left(L_{2}\right)$ with $\|\varphi\|\left\|\varphi^{-1}\right\|<2$, then $L_{1}$ and $L_{2}$ are homeomorphic (here $C_{0}\left(L_{1}\right)$ and $C_{0}\left(L_{2}\right)$ are complex spaces). Properties of extremely regular function spaces were studied in [8].

In this paper, we demonstrate that extremely regular function spaces play a role in a quite recent theory of Banach spaces, namely that involving Daugavet spaces, diameter 2 spaces, and octahedral spaces. Let us briefly explain some main lines of this theory before returning to extremely regular function spaces and our results.

Let $X$ be a Banach space and $B_{X}$ its unit ball. By a slice of $B_{X}$ we mean a set of the form $S\left(x^{*}, \varepsilon\right):=\left\{x \in B_{X}: \operatorname{Re} x^{*}(x)>1-\varepsilon\right\}$, where $x^{*}$ is in the unit sphere $S_{X^{*}}$ of $X^{*}$ and $\varepsilon>0$. A finite convex combination of slices

2010 Mathematics Subject Classification. 46B20; 46B22.

Key words and phrases. Extremely regular function space, strong diameter 2 property, almost square space, octahedrality, Daugavet property.

Research by the third-named author supported, in part, by institutional research funding IUT20-57 of the Estonian Ministry of Education and Research. 
of $B_{X}$ is a set $S$ of the form $S=\sum_{i=1}^{n} \lambda_{i} S\left(x_{i}^{*}, \varepsilon_{i}\right)$ where $n \in \mathbb{N}, \lambda_{i}>0$, $\sum_{i=1}^{n} \lambda_{i}=1, x_{i}^{*} \in S_{X^{*}}$, and $\varepsilon_{i}>0$.

Definition 1.2. A Banach space $X$ has the strong diameter 2 property (briefly, $S D 2 P$ ) if every finite convex combination of slices of $B_{X}$ has diameter 2 .

A lemma by Bourgain [10, page 26, Lemma II.1] says that every nonempty relatively weakly open subset of $B_{X}$ contains a finite convex combination of slices. Thus the SD2P implies that every non-empty relatively weakly open subset of $B_{X}$ has diameter 2, which in turn implies that every slice of $B_{X}$ has diameter 2. None of these implications is reversible ([5], [12]).

It is an important observation of Deville and Godefroy from the late 1980s, stated without proof in [11, that $X$ having SD2P is equivalent to $X^{*}$ being octahedral. A Banach space $Z$ is octahedral if, for every finite-dimensional subspace $F$ of $Z$ and every $\varepsilon>0$, there exists a $y \in S_{Z}$ such that

$$
\|x+t y\| \geq(1-\epsilon)(\|x\|+|t|) \quad \text { for every } x \in F \text { and every } t \in \mathbb{K} .
$$

A complete proof of this equivalence can be found in [4, Corollary 2.2] (the proof is carried out for the real case, but it is not too hard to see that the result holds also in the complex case).

Definition 1.3. A Banach space $X$

(1) is almost square (briefly, $A S Q$ ) if whenever $n \in \mathbb{N}$ and $x_{1}, \ldots, x_{n} \in$ $S_{X}$, there exists a sequence $\left(y_{k}\right)$ in $B_{X}$ such that $\left\|x_{i} \pm y_{k}\right\| \underset{k \rightarrow \infty}{\longrightarrow} 1$ for every $i \in\{1, \ldots, n\}$ and $\left\|y_{k}\right\| \underset{k \rightarrow \infty}{\longrightarrow} 1$.

(2) has the symmetric strong diameter 2 property (briefly, SSD2P) if whenever $n \in \mathbb{N}, S_{1}, \ldots, S_{n}$ are slices of $B_{X}$, and $\varepsilon>0$, there exist $x_{i} \in S_{i}, i=1, \ldots, n$, and $y \in B_{X}$ such that $x_{i} \pm y \in S_{i}$ for every $i \in\{1, \ldots, n\}$ and $\|y\|>1-\varepsilon$.

ASQ Banach spaces were studied in [1]. The SSD2P has not been fully explored yet, but can be found in [2, Lemma 4.1], where it is observed that the SD2P is implied by the SSD2P. In turn, it is not too hard to show that ASQ Banach spaces have the SSD2P. On the other hand, the space $L_{1}[0,1]$ has the SD2P, but not the SSD2P, and the space $C[0,1]$ has the SSD2P, but is not ASQ.

The property of a Banach space to be ASQ - and also the SSD2P - is rather strong. The widest class of spaces known to be ASQ are non-reflexive $M$-embedded spaces [1, Corollary 4.3]. Also, $c_{0}(X)$ is ASQ for any Banach space $X$. The widest class of spaces known to have the SSD2P are uniform algebras [2, Theorem 4.2]. Also, $\ell_{\infty}(X)$ has the SSD2P for any Banach space $X$.

Let us also relate the Daugavet property to the diameter 2 properties. Recall that a bounded linear operator $T$ on a Banach space $X$ is said to satisfy the Daugavet equation if $\|I+T\|=1+\|T\|$. Daugavet himself discovered in [9] that every compact operator on $C[0,1]$ satisfies this equation, thus initiating a very important topic in the theory of Banach spaces. In [14, Lozanovskiu obtained the analogous result for $L_{1}[0,1]$. 
Definition 1.4. A Banach space $X$ has the Daugavet property if every rank 1 operator on $X$ satisfies the Daugavet equation.

Note that if a Banach space $X$ has the Daugavet property, then, in fact, every weakly compact operator on $X$ enjoys the Daugavet equation (see, e.g., [13, Theorem 2.3] or [17, Theorem 2.7]).

Towards the end of the 1990s, the Daugavet property was described in geometrical terms (see [13, Lemmas 2.1 and 2.2], [16, Lemmas 2 and 3], and [17. Lemmas 2.2-2.4]). Spaces with the Daugavet property have the SD2P [2, Theorem 4.4] and are octahedral [4, Corollary 2.5].

Finally, we can announce our main results: An extremely regular subspace of $C_{0}(L)$

- has the SSD2P (Theorem 2.2);

- is ASQ whenever L is non-compact (Theorem 2.5);

- has the Daugavet property whenever $L$ does not contain isolated points (Theorem 2.6);

- contains an $\varepsilon$-isometric copy of $c_{0}$ whenever $0<\varepsilon<1$ (Theorem 3.1).

In fact, we prove these results for a wider class of subspaces of $C_{0}(L)$ than extremely regular ones, that we call somewhat regular subspaces (see Definition 2.1 below).

Throughout the paper, it should not make any confusion to denote, for a functional $\mu \in C_{0}(L)^{*}$, its representing (regular) Borel measure also by $\mu$.

\section{Diameter 2 properties for subspaces of $C_{0}(L)$}

Definition 2.1. We call a linear subspace $\mathcal{A}$ of $C_{0}(L)$ somewhat regular, if, whenever $V$ is a non-empty open subset of $L$ and $0<\varepsilon<1$, there is an $f \in \mathcal{A}$ such that

$$
\|f\|=1 \quad \text { and } \quad|f(x)| \leq \varepsilon \text { for every } x \in L \backslash V .
$$

Notice that, in this case, $\left|f\left(x_{0}\right)\right|=1$ for some $x_{0} \in V$, thus one may choose an $f \in \mathcal{A}$ satisfying (2.1) so that $f\left(x_{0}\right)=1$ for some $x_{0} \in V$.

It is clear that extremely regular subspaces of $C_{0}(L)$ are somewhat regular. On the other hand, whenever $x_{0}$ is an accumulation point of $L$, the subspace $\left\{f \in C_{0}(L): f\left(x_{0}\right)=0\right\}$ of $C_{0}(L)$ is somewhat regular by courtesy of Urysohn's lemma, but fails to be extremely regular. Thus the class of somewhat regular subspaces of $C_{0}(L)$ is strictly larger than that of extremely regular ones.

Theorem 2.2. Somewhat regular linear subspaces of $C_{0}(L)$ have the $S S D 2 P$.

Theorem 2.2 is a corollary from the following lemma.

Lemma 2.3. Let $\mathcal{A}$ be a somewhat regular linear subspace of $C_{0}(L)$, and let $n, m \in \mathbb{N}, f_{1}, \ldots, f_{n} \in B_{\mathcal{A}}, \mu_{1}, \ldots, \mu_{m} \in B_{C_{0}(L)^{*}}$, and $\varepsilon>0$. Then there are $g_{1}, \ldots, g_{n}, \phi \in B_{\mathcal{A}}$ such that, for every $j \in\{1, \ldots, n\}$,

(1) $\left|\mu_{i}\left(f_{j}-g_{j}\right)\right|<\varepsilon$ for every $i \in\{1, \ldots, m\}$;

(2) $\left|\mu_{i}(\phi)\right|<\varepsilon$ for every $i \in\{1, \ldots, m\}$;

(3) $\|\phi\|>1-\varepsilon$;

(4) $\left\|g_{j} \pm \phi\right\| \leq 1$. 
When dealing with subspaces of $C_{0}(L)$, the main challenge is often to find a substitute for Urysohn's lemma (see, e.g., 6, Section 2]). The following lemma - which the proofs of both Lemma 2.3 and Theorem 2.6 below rely on-is a "Urysohn's lemma" for somewhat regular subspaces of $C_{0}(L)$. The lemma is inspired by [15, proof of Theorem 1].

Lemma 2.4 (cf. [15, proof of Theorem 1]). Let $\mathcal{A}$ be a somewhat regular linear subspace of $C_{0}(L)$, let $V$ be a non-empty open subset of $L$, and let $0<\varepsilon<1$. Then there are an $x_{0} \in V$ and an $f \in \mathcal{A}$ such that

(1) $f\left(x_{0}\right)=1 \leq\|f\| \leq 1+\varepsilon$;

(2) $|1-f(x)| \leq 1+\varepsilon$ for every $x \in V$;

(3) $|f(x)| \leq \varepsilon$ for every $x \in L \backslash V$.

Proof. Let $0<\delta<1$ and let $n \in \mathbb{N}$ satisfy $2 / n<\delta$. Putting $V_{0}:=V$, by courtesy of the somewhat regularity of $\mathcal{A}$, one can recursively find points $x_{1}, \ldots, x_{n} \in V$, functions $g_{1}, \ldots, g_{n} \in \mathcal{A}$, and nonvoid open subsets $V_{0} \supset$ $V_{1} \supset \cdots \supset V_{n}$ such that, for every $j \in\{1, \ldots, n\}$,

$$
x_{j} \in V_{j-1}, \quad g_{j}\left(x_{j}\right)=\left\|g_{j}\right\|=1, \quad\left|g_{j}(x)\right| \leq \delta \text { for every } x \in L \backslash V_{j-1},
$$

and $V_{j}=\left\{x \in V_{j-1}:\left|g_{j}(x)-1\right|<\delta\right\}$; thus, in fact, $x_{j} \in V_{j}$. Defining $x_{0}:=x_{n}$ and

$$
g:=\frac{g_{1}+\cdots+g_{n}}{n},
$$

one has $\|g\| \leq 1,|g(x)| \leq \delta$ for every $x \in L \backslash V$, and

$$
|1-g(x)| \leq \frac{1}{n} \sum_{j=1}^{n}\left|1-g_{j}(x)\right| \quad \text { for every } x \in L .
$$

Now let $x \in V$. Put $k:=\max \left\{j \in\{0,1, \ldots, n\}: x \in V_{j}\right\}$. For $1 \leq j \leq k$, one has $\left|1-g_{j}(x)\right|<\delta ; \delta \leq\left|1-g_{k+1}(x)\right| \leq 2$; and, for $k+2 \leq j \leq n$, one has $\left|g_{j}(x)\right| \leq \delta$ and hence $\left|1-g_{j}(x)\right| \leq 1+\delta$. Thus

$$
|1-g(x)| \leq \frac{(n-1)(1+\delta)+2}{n}<1+\delta+\frac{2}{n}<1+2 \delta .
$$

Since $x_{0}=x_{n} \in V_{n}$, one has $\left|g_{j}\left(x_{0}\right)-1\right|<\delta$ for every $j \in\{1, \ldots, n\}$ and thus $\left|g\left(x_{0}\right)-1\right|<\delta$. Defining $f:=\left(1 / g\left(x_{0}\right)\right) g$, it remains to observe that, taking, from the very beginning, $\delta$ to be "small enough", the conditions (1)-(3) obtain, because, since $\left|g\left(x_{0}\right)\right|>1-\delta$,

$$
1=f\left(x_{0}\right) \leq\|f\|=\frac{\|g\|}{\left|g\left(x_{0}\right)\right|}<\frac{1}{1-\delta},
$$

for every $x \in V$,

$$
|1-f(x)|=\frac{\left|g\left(x_{0}\right)-g(x)\right|}{\left|g\left(x_{0}\right)\right|}<\frac{\left|g\left(x_{0}\right)-1\right|+|1-g(x)|}{1-\delta}<\frac{1+3 \delta}{1-\delta},
$$

and, for every $x \in L \backslash V$,

$$
|f(x)|=\frac{|g(x)|}{\left|g\left(x_{0}\right)\right|}<\frac{\delta}{1-\delta} .
$$


Proof of Lemma 2.3. Let $0<\delta<1 / 2$. Since $L$ is infinite, there is a point $y \in L$ such that $\max _{1 \leq i \leq m}\left|\mu_{i}\right|(\{y\})<\delta$; hence, by the regularity of $\mu_{1}, \ldots, \mu_{m}$ and the continuity of $f_{1} \ldots, f_{n}$, there is a non-empty open subset $V$ of $L$ such that

$$
\max _{1 \leq i \leq m}\left|\mu_{i}\right|(V)<\delta \quad \text { and } \max _{1 \leq j \leq n} \sup _{x, z \in V}\left|f_{j}(x)-f_{j}(z)\right|<\delta .
$$

Since, by our assumption, $\mathcal{A}$ is somewhat regular, there are $x_{0} \in V$ and $f \in \mathcal{A}$ satisfying the conditions (1)-(3) of Lemma 2.4 with $\varepsilon$ replaced by $\delta$. For every $j \in\{1, \ldots, n\}$, defining $\alpha_{j}:=f_{j}\left(x_{0}\right)$ and $h_{j}:=f_{j}-\alpha_{j} f \in \mathcal{A}$, one has $h_{j}\left(x_{0}\right)=0$ and $\left\|h_{j}\right\| \leq 1+2 \delta$, because

$$
\left|h_{j}(x)\right| \leq \begin{cases}\left|f_{j}(x)-\alpha_{j}\right|+\left|\alpha_{j}\right||1-f(x)| \leq 1+2 \delta, & \text { if } x \in V ; \\ \left|f_{j}(x)\right|+\left|\alpha_{j}\right||f(x)| \leq 1+\delta, & \text { if } x \in L \backslash V .\end{cases}
$$

For every $j \in\{1, \ldots, n\}$, defining $g_{j}:=(1-2 \delta) h_{j}$, one has $\left\|g_{j}\right\| \leq 1-4 \delta^{2}$ and, for every $i \in\{1, \ldots, m\}$, since

$$
\left|\mu_{i}(f)\right| \leq \int_{L \backslash V}|f| d\left|\mu_{i}\right|+\int_{V}|f| d\left|\mu_{i}\right| \leq \delta\left|\mu_{i}\right|(L \backslash V)+2\left|\mu_{i}\right|(V)<3 \delta,
$$

also

$$
\left|\mu_{i}\left(f_{j}-g_{j}\right)\right| \leq 2 \delta\left|\mu_{i}\left(f_{j}\right)\right|+(1-2 \delta)\left|\alpha_{j}\right|\left|\mu_{i}(f)\right|<5 \delta .
$$

Choose an open neighbourhood $U \subset V$ of $x_{0}$ such that

$$
\max _{1 \leq j \leq n} \sup _{x \in U}\left|g_{j}(x)\right| \leq \delta .
$$

Since $\mathcal{A}$ is somewhat regular, there is a $\psi \in \mathcal{A}$ such that

$$
\|\psi\|=1 \quad \text { and } \quad|\psi(x)| \leq 4 \delta^{2} \text { for every } x \in L \backslash U .
$$

Put $\phi:=(1-\delta) \psi$. Then, for every $j \in\{1, \ldots, m\}$, one has $\left\|g_{j} \pm \phi\right\| \leq 1$, i.e., the condition (4) holds, and, for every $i \in\{1, \ldots, m\}$,

$$
\left|\mu_{i}(\phi)\right| \leq \int_{L \backslash V}|\phi| d\left|\mu_{i}\right|+\int_{V}|\phi| d\left|\mu_{i}\right| \leq 4 \delta^{2}\left|\mu_{i}\right|(L \backslash V)+\left|\mu_{i}\right|(V)<5 \delta .
$$

Thus, one observes that taking, from the very beginning, $\delta$ to be "small enough", also the conditions (1)-(3) hold.

If the space $L$ is non-compact, a stronger statement than that of Theorem 2.2 is true.

Theorem 2.5. Assume that $L$ is non-compact. Then every somewhat regular linear subspace of $C_{0}(L)$ is $A S Q$.

Proof. Let $\mathcal{A}$ be a somewhat regular linear subspace of $C_{0}(L)$, and let $n \in \mathbb{N}$, $f_{1}, \ldots, f_{n} \in S_{\mathcal{A}}$, and $\varepsilon>0$. It suffices to find an $f \in \mathcal{A}$ such that $\|f\|=1$ and

$$
\left\|f_{j} \pm f\right\| \leq 1+\varepsilon \quad \text { for every } j \in\{1, \ldots, n\} .
$$

To this end, observe that the sets $K_{j}:=\left\{x \in L:\left|f_{j}(x)\right| \geq \varepsilon\right\}, j=1, \ldots, n$, are compact; thus also their union $K:=\bigcup_{j=1}^{n} K_{j}$ is compact, and its complement $V:=L \backslash K$ is non-empty and open. By the somewhat regularity of $\mathcal{A}$, there is an $f \in \mathcal{A}$ satisfying (2.1). This $f$ also satisfies (2.2). 
Our next result produces examples of spaces with the Daugavet property.

Theorem 2.6. Assume that $L$ does not contain isolated points. Then every somewhat regular linear subspace of $C_{0}(L)$ has the Daugavet property.

Proof. Let $\mathcal{A}$ be a somewhat regular linear subspace of $C_{0}(L)$, let $g \in S_{\mathcal{A}}$, let $\mu \in S_{C_{0}(L)^{*}}$ be such that $\left\|\left.\mu\right|_{\mathcal{A}}\right\|=1$, and let $\alpha, \varepsilon>0$. In order for $\mathcal{A}$ to have the Daugavet property, by [17, Lemma 2.2] (or [13, Lemma 2.2]), it suffices to find a $\psi \in S_{\mathcal{A}}$ satisfying

$$
\operatorname{Re} \mu(\psi)>1-\alpha \text { and }\|g+\psi\|>2-\varepsilon .
$$

To this end, let $\delta \in(0,1 / 3)$, let $\phi \in S_{\mathcal{A}}$ be such that $\operatorname{Re} \mu(\phi)>1-\delta$, let $y_{0} \in L$ be such that $\left|g\left(y_{0}\right)\right|=1$, and let an open neighbourhood $U$ of $y_{0}$ be such that

$$
\left|g(x)-g\left(y_{0}\right)\right|<\delta \quad \text { and } \quad\left|\phi(x)-\phi\left(y_{0}\right)\right|<\delta \quad \text { for all } x \in U .
$$

Since $y_{0}$ is not an isolated point, the set $U$ is infinite; thus there is a point $z_{0} \in U$ such that $|\mu|\left(\left\{z_{0}\right\}\right)<\delta$. By the regularity of $\mu$, there is an open neighbourhood $V$ of $z_{0}$ such that $|\mu|(V)<\delta$. One may assume that $V \subset U$ and thus

$$
|\phi(x)-\phi(z)|<2 \delta \quad \text { for all } x, z \in V .
$$

Since $\mathcal{A}$ is somewhat regular, there are $x_{0} \in V$ and $f \in \mathcal{A}$ satisfying the conditions (1)-(3) of Lemma 2.4 with $\varepsilon$ replaced by $\delta$. Put $h:=\phi-\phi\left(x_{0}\right) f$; then $h\left(x_{0}\right)=0$ and $\|h\| \leq 1+3 \delta$ (this can be shown as in the proof of Theorem 2.2 for $\left.h_{j}\right)$; indeed,

$$
|h(x)| \leq \begin{cases}\left|\phi(x)-\phi\left(x_{0}\right)\right|+\left|\phi\left(x_{0}\right)\right||1-f(x)| \leq 1+3 \delta, & \text { if } x \in V ; \\ |\phi(x)|+\left|\phi\left(x_{0}\right)\right||f(x)| \leq 1+\delta, & \text { if } x \in L \backslash V .\end{cases}
$$

Since $h\left(x_{0}\right)=0$, there is an open neighbourhood $W$ of $x_{0}$ such that

$$
|h(x)|<\delta \quad \text { for all } x \in W .
$$

One may assume that $W \subset V$. Since $\mathcal{A}$ is somewhat regular, there are $w_{0} \in W$ and $\widehat{f} \in \mathcal{A}$ such that

$$
\widehat{f}\left(w_{0}\right)=\|\widehat{f}\|=1 \quad \text { and } \quad|\widehat{f}(x)| \leq \delta \quad \text { for every } x \in L \backslash W .
$$

Putting $\widehat{\psi}:=h+g\left(w_{0}\right) \widehat{f}$, one has $\|\widehat{\psi}\| \leq 1+4 \delta$, because

$$
|\widehat{\psi}(x)| \leq|h(x)|+|\widehat{f}(x)| \leq \begin{cases}\delta+1, & \text { if } x \in W \\ (1+3 \delta)+\delta=1+4 \delta, & \text { if } x \in L \backslash W .\end{cases}
$$

Since

$$
\begin{aligned}
\|\widehat{\psi}+g\| & \geq\left|\widehat{\psi}\left(w_{0}\right)+g\left(w_{0}\right)\right| \geq 2\left|g\left(w_{0}\right)\right|-\left|h\left(w_{0}\right)\right| \\
& \geq 2\left|g\left(y_{0}\right)\right|-2\left|g\left(y_{0}\right)-g\left(w_{0}\right)\right|-\left|h\left(w_{0}\right)\right| \\
& >2-2 \delta-\delta=2-3 \delta
\end{aligned}
$$

one has $\|\widehat{\psi}\|>1-3 \delta$; thus, for $\psi:=\widehat{\psi} /\|\widehat{\psi}\|$, one has

$$
\|\widehat{\psi}-\psi\|=\left|1-\frac{1}{\|\widehat{\psi}\|}\right|\|\widehat{\psi}\|=|\|\widehat{\psi}\|-1| \leq 4 \delta,
$$


and hence

$$
\|g+\psi\| \geq\|g+\widehat{\psi}\|-\|\widehat{\psi}-\psi\|>2-3 \delta-4 \delta=2-7 \delta .
$$

One has

$$
\begin{aligned}
\operatorname{Re} \mu(\widehat{\psi}) & =\operatorname{Re} \mu(h)+\operatorname{Re} g\left(w_{0}\right) \mu(\widehat{f})=\operatorname{Re} \mu(\phi)-\operatorname{Re} \phi\left(x_{0}\right) \mu(f)+\operatorname{Re} g\left(w_{0}\right) \mu(\widehat{f}) \\
& >1-\delta-|\mu(f)|-|\mu(\widehat{f})| .
\end{aligned}
$$

Since

$$
\begin{aligned}
|\mu(f)| & \leq\left|\int_{L} f d \mu\right| \leq \int_{L}|f| d|\mu|=\int_{V}|f| d|\mu|+\int_{L \backslash V}|f| d|\mu| \\
& \leq(1+\delta)|\mu|(V)+\delta|\mu|(L \backslash V)<(1+\delta) \delta+\delta=(2+\delta) \delta<3 \delta,
\end{aligned}
$$

and, similarly, $|\mu(\widehat{f})|<2 \delta$, it follows that $\operatorname{Re} \mu(\widehat{\psi})>1-6 \delta$, and thus

$$
\operatorname{Re} \mu(\psi)=\frac{\operatorname{Re} \mu(\widehat{\psi})}{\|\widehat{\psi}\|} \geq \frac{\operatorname{Re} \mu(\widehat{\psi})}{1+4 \delta}>\frac{1-6 \delta}{1+4 \delta} .
$$

Hence one observes that, choosing, from the very beginning, $\delta$ to be "small enough", the function $\psi$ meets the conditions (2.3).

\section{Containment of $c_{0}$ And $\ell_{1}$}

Let $X$ and $Y$ be normed spaces, and let $0<\varepsilon<1$. Recall that a linear surjection $T: X \rightarrow Y$ is called an $\varepsilon$-isometry if

$$
(1-\varepsilon)\|x\| \leq\|T x\| \leq(1+\varepsilon)\|x\| \quad \text { for every } x \in X .
$$

It is well known that $C_{0}(L)$ contains isometric copies of $c_{0}$ (see e.g [3, Proposition 4.3.11]), and the same is true for many of its subspaces. For the somewhat regular linear subspaces of $C_{0}(L)$ we have the following theorem.

Theorem 3.1. Let $\mathcal{A}$ be a somewhat regular closed linear subspace of $C_{0}(L)$. Then, whenever $0<\varepsilon<1$, there is an $\varepsilon$-isometry from $c_{0}$ onto a closed linear subspace of $\mathcal{A}$.

Proof. Let $0<\varepsilon<1$. Choose pairwise disjoint nonvoid open subsets $U_{j}$, $j \in \mathbb{N}$, of $L$. Since $\mathcal{A}$ is somewhat regular, for every $j \in \mathbb{N}$, there are an $x_{j} \in U_{j}$ and an $f_{j} \in \mathcal{A}$ such that

$$
f_{j}\left(x_{j}\right)=1 \quad \text { and } \quad\left|f_{j}(x)\right| \leq \frac{\varepsilon}{2^{j}} \quad \text { for every } x \in L \backslash V_{j} .
$$

Denoting by $c_{00}$ the linear subspace of finitely supported sequences in $c_{0}$, let $S_{0}: c_{00} \rightarrow \mathcal{A}$ be the linear operator satisfying $S_{0} e_{j}=f_{j}$ for every $j \in \mathbb{N}$ where $e_{j}$ are the standard unit vectors in $c_{0}$. Observing that, whenever $a=\sum_{j=1}^{n} \alpha_{j} e_{j} \in S_{c_{00}}$ and $x \in L$, one has

$$
\left|\left(S_{0} a\right)(x)\right|=\left|\sum_{j=1}^{n} \alpha_{j} f_{j}(x)\right| \leq \sum_{j=1}^{n}\left|f_{j}(x)\right| \leq 1+\sum_{j=1}^{n} \frac{\varepsilon}{2^{j}}<1+\varepsilon
$$

(because $\left|f_{j}(x)\right| \leq \varepsilon / 2^{j}$ whenever $x \notin U_{j}$, and there is at most one $j \in \mathbb{N}$ such that $x \in U_{j}$ (in which case $\left|f_{j}(x)\right| \leq 1$ )), thus $S_{0}$ is bounded and $\left\|S_{0}\right\| \leq 1+\varepsilon$. Letting $S: c_{0} \rightarrow \mathcal{A}$ be the bounded linear extension of $S_{0}$, 
one has $\|S\| \leq 1+\varepsilon$ as well, and it remains to observe that, whenever $a=\left(\alpha_{j}\right)_{j=1}^{\infty} \in c_{0}$, picking $k \in \mathbb{N}$ such that $\left|\alpha_{k}\right|=\|a\|$, one has

$$
\begin{aligned}
\|S a\| & =\left\|\sum_{j=1}^{\infty} \alpha_{j} f_{j}\right\| \geq\left|\sum_{j=1}^{\infty} \alpha_{j} f_{j}\left(x_{k}\right)\right| \geq\left|\alpha_{k}\right|\left|f_{k}\left(x_{k}\right)\right|-\sum_{\substack{j=1 \\
j \neq k}}^{\infty}\left|\alpha_{j}\right|\left|f_{j}\left(x_{k}\right)\right| \\
& \geq\|a\|-\|a\| \sum_{j=1}^{\infty} \frac{\varepsilon}{2^{j}}=(1-\varepsilon)\|a\|,
\end{aligned}
$$

because, for $j \neq k$, one has $x_{k} \notin V_{j}$ and thus $\left|f_{j}\left(x_{k}\right)\right| \leq \varepsilon$.

It is natural to ask about containment of $\ell_{1}$ in somewhat regular linear subspaces of $C_{0}(L)$. If $L$ does not contain isolated points, we have from Theorem [2.6 and [13, Theorem 2.9] that all somewhat regular linear subspaces of $C_{0}(L)$ contain $\ell_{1}$ (even asymptotically isometric copies of $\left.\ell_{1}\right)$. But, if $L$ contains isolated points, the picture is not so clear. In this case there might be somewhat regular subspaces of $C_{0}(L)$ which contain $\ell_{1}$ and other such subspaces which do not. For an example, take $C(\beta \mathbb{N})$ and its subspaces $X=\{f \in C(\beta \mathbb{N}): f(x)=0$ for every $x \in \beta \mathbb{N} \backslash \mathbb{N}\}$ and $Y=\{f \in C(\beta \mathbb{N}): f(y)=0\}$ where $y \in \beta \mathbb{N} \backslash \mathbb{N}$ is a fixed element. It is straightforward to show that both these subspaces are somewhat regular. Moreover, $X$ is isometrically isomorphic to $c_{0}$ and $Y$ is isomorphic to $C(\beta \mathbb{N})$.

\section{REFERENCES}

[1] T. A. Abrahamsen, J. Langemets, and V. Lima, Almost square Banach spaces, J. Math. Anal. Appl. 434 (2016), no. 2, 1549-1565. MR 3415738

[2] T. A. Abrahamsen, V. Lima, and O. Nygaard, Remarks on diameter 2 properties, J. Convex. Anal. 20 (2013), no. 2, 439-452. MR 3098474

[3] F. Albiac and N. J. Kalton, Topics in Banach space theory, Graduate Texts in Mathematics, vol. 233, Springer, New York, 2006. MR 2192298 (2006h:46005)

[4] J. Becerra Guerrero, G. López Pérez, and A. Rueda Zoca, Octahedral norms and convex combination of slices in Banach spaces, J. Funct. Anal. 266 (2014), no. 4, 2424-2435. MR 3150166

[5] J. Becerra Guerrero, G. López Pérez, and A. Rueda Zoca, Big slices versus big relatively weakly open subsets in Banach spaces, J. Math. Anal. Appl. 428 (2015), no. 2, 855-865. MR 3334951

[6] B. Cascales, A. J. Guirao, and V. Kadets, A Bishop-Phelps-Bollobás type theorem for uniform algebras, Adv. Math. 240 (2013), 370-382. MR 3046314

[7] B. Cengiz, A generalization of the Banach-Stone theorem, Proc. Amer. Math. Soc. 40 (1973), no. 2, 426-430. MR 0320723 (47 $\sharp 9258)$

[8] B. Cengiz, On extremely regular function spaces, Pacific J. Math., 49 (1973), no. 2 , 335-338. MR $0358317((50 \sharp 10783))$

[9] I. K. Daugavet, A property of completely continuous operators in the space $C$ (Russian), Uspehi Mat. Nauk 18 (1963), no. 5 (113), 157-158. MR 0157225 (28 $\sharp 461)$

[10] N. Ghoussoub, G. Godefroy, B. Maurey, and W. Schachermayer, Some topological and geometrical structures in Banach spaces, Mem. Amer. Math. Soc. 70 (1987), no. 378, iv+116 pp. MR 0912637 (89h:46024)

[11] G. Godefroy, Metric characterization of first Baire class linear forms and octahedral norms, Studia Math. 95 (1989), no. 1, 1-15. MR 1024271 (91h:46020)

[12] R. Haller and J. Langemets, Two remarks on diameter 2 properties, Proc. Est. Acad. Sci. 63 (2014), no. 1, 2-7. 
[13] V. M. Kadets, R. V. Shvidkoy, G. G. Sirotkin, and D. Werner, Banach spaces with the Daugavet property, Trans. Amer. Math. Soc. 352 (2000), no. 2, 855-873. MR 1621757 (2000c:46023)

[14] G. Ya. Lozanovskiǔ, On almost integral operators in KB-spaces (Russian. English summary), Vestnik Leningrad. Univ. 21 (1966), no. 7, 35-44. MR 0208375 (34 $\sharp 8185)$

[15] O. Nygaard and D. Werner, Slices in the unit ball of a uniform algebra, Arch. Math. (Basel) 76 (2001), no. 6, 441-444. MR 1831500 (2002e:46057)

[16] R. V. Shvydkoy, Geometric aspects of the Daugavet property, J. Funct. Anal. 176 (2000), no. 2, 198-212. MR 1784413 (2001h:46019)

[17] D. Werner, Recent progress on the Daugavet property, Irish Math. Soc. Bull. (2001), no. 46, 77-97. MR 1856978 (2002i:46014)

Department of Mathematics, University of Agder, Servicebox 422, 4604 KRISTIANSAND, NORWAY.

E-mail address: Trond.A.Abrahamsen@uia.no

$U R L:$ http://home.uia.no/trondaa/index.php3

E-mail address: Olav.Nygaard@uia.no

URL: http://home.uia.no/olavn/

Institute of Mathematics and Statistics, University of TARtu, J. Liivi 2 , 50409 TARTU, ESTONIA

E-mail address: mart.poldvere@ut.ee 